\title{
Analysis of Current Status on a New Public Health Nutrition Service Pattern in China: A Nutrition Outpatient Clinic-Based Study
}

\author{
Yunpeng Jin ${ }^{1}$ and Xiaolin $\mathrm{Li} \mathbb{D}^{2}$ \\ ${ }^{1}$ Division of Cardiology, The Fourth Affiliated Hospital of Zhejiang University School of Medicine, N1 Shangcheng Road, \\ Yiwu 322000, Zhejiang, China \\ ${ }^{2}$ Division of Nutrition, The Fourth Affiliated Hospital of Zhejiang University School of Medicine, N1 Shangcheng Road, \\ Yiwu 322000, Zhejiang, China
}

Correspondence should be addressed to Xiaolin Li; 8617194@zju.edu.cn

Received 15 June 2018; Revised 29 July 2018; Accepted 6 August 2018; Published 9 August 2018

Academic Editor: Abdelaziz M. Thabet

Copyright (C) 2018 Yunpeng Jin and Xiaolin Li. This is an open access article distributed under the Creative Commons Attribution License, which permits unrestricted use, distribution, and reproduction in any medium, provided the original work is properly cited.

\begin{abstract}
Background. Nutrition outpatient clinics were launched in some hospitals as a new pattern of public health nutrition service in recent years in China. The aim of this study was to review and analysis demographics and consultation spectrum in a single nutrition outpatient clinical center in China. Methods. The retrospective study was performed in the nutrition outpatient clinical center launched by a comprehensive teaching hospital in Yiwu, Zhejiang Province (China). 1014 patients attending the clinic from August 2015 to February 2018 were included. The clinical records including relevant history and baseline data were reviewed and analyzed. Results. Majority of the patients (41.9\%) came to our clinical center for nutrition consultation of healthy dietary services, $32.1 \%$ for malnutrition, $6.7 \%$ for diabetes, $6.3 \%$ for neoplasms, $5.3 \%$ for digestive system diseases, and the last $7.6 \%$ for hypertension, hematologic diseases, thyroid diseases, and so on. More minor patients came for healthy dietary services compared with the population $(\mathrm{P}<0.001)$, and, on the contrary, more adult patients came for malnutrition service, especially obesity $(\mathrm{P}<0.05)$, while more elderly patients came for consultation services of diabetes, neoplasms, digestive system diseases, and hypertension $(\mathrm{P}<0.05)$. Conclusion. Our study provided important data of the new public health nutrition service pattern in China which has never been reported yet. It indicated the huge demand of nutrition outpatient clinic, especially nutrition consultation services of healthy diet and malnutrition; further studies about the validity of the new pattern in improving public health nutrition status are expected in the future.
\end{abstract}

\section{Introduction}

More and more nutrition health issues occurred in China recent years as economy develops. The Fourth National Nutrition and Health Survey showed that China is facing the dual challenges of nutritional deficiencies and overnutrition $[1,2]$. The dramatic increase in nutrition-related chronic diseases such as obesity, hyperlipidemia, diabetes, cardiocerebral vascular disease, and cancer posed a heavy burden on society $[3,4]$. The demand of public health nutrition services has increased in China. Therefore, nutrition outpatient clinics were launched in some hospitals in recent years.
As a new pattern of public health nutrition service, the nutrition outpatient clinics in China were supported by nutrition physicians, not only physicians but also nutritionists $[5,6]$. According to several investigation researches in China, about $12 \%$ of Chinese hospitals have launched nutrition outpatient clinics. However, the data about the nutrition outpatient clinics has never been reported yet.

In our nutrition outpatient clinical center, thousands of patients have been attended in the past more than two years. The aim of this study was to review and analyze demographics and consultation spectrum in a single nutrition outpatient clinical center in China. 


\section{Methods}

2.1. Study Subjects. All consecutive new patients seen in a comprehensive teaching hospital (The Fourth Affiliated Hospital of Zhejiang University School of Medicine) nutrition clinical center from August 2015 to February 2018 were included in this retrospective study. The clinical records were reviewed and the demographics of the patients, their height, weight, Body Mass Index (BMI), and the cause of attending were analyzed. According to the types of nutrition-related issues, nutrition services included healthy dietary services, malnutrition, diabetes, neoplasms, digestive system diseases, hypertension, and so on.

2.2. Ethical Aspects. Ethical approval for publication of this data was obtained from the Ethics Committee of The Fourth Affiliated Hospital of Zhejiang University School of Medicine.

2.3. Anthropometric Measurements. All patients used the same devices, which were calibrated at the time of measurement. The height and weight of adolescents were measured with thin clothes (shorts and T-shirts) and without shoes. Height was measured to the nearest $0.1 \mathrm{~cm}$ with a freestanding stadiometer mounted on a rigid tripod (GMCSI, Xindong Huateng Sports Equipment Co. Ltd., Beijing, China). Fasting body weight was measured to the nearest $0.1 \mathrm{~kg}$ on a digital scale (RGT-140, Weighing Apparatus Co. Ltd, Changzhou Wujin, China). Body mass index (BMI) was calculated by body weight $(\mathrm{kg}) /$ [body height $(\mathrm{m})]^{2}$. Thinness, overweight, and obesity were defined according to the special cutoffs of $\mathrm{BMI}<18.5 \mathrm{~kg} / \mathrm{m}^{2}, \mathrm{BMI} \geq 24 \mathrm{~kg} / \mathrm{m}^{2}$, and $\mathrm{BMI} \geq 28 \mathrm{~kg} / \mathrm{m}^{2}$, respectively, for the Chinese adult population [7]. Thinness, overweight, and obesity in children and adolescents population were defined according to age- and sex-specific BMI cutoffs developed for Chinese children and adolescents [8]. Malnutrition was defined by BMI z-score in infancy, related to the 2006 World Health Organization growth references.

2.4. Statistical Analysis. All categorical variables are expressed as proportions. Chi-square tests were used for intergroup comparisons of categorical variables. A p value $<0.05$ was considered significant, and all $\mathrm{p}$ values are 2sided. Statistical analyses were performed with SPSS software version 19 (IBM/SPSS, Armonk, NY USA 2012).

\section{Results}

From August 2015 to February 2018, 1014 patients had been seen in the nutrition clinical center as new cases. The age ranged from 4 months to 92 years old. Proportion of female patients was a little bit more than male. A large proportion of adult patients (18-64 years old) came to our clinic for nutrition services, and minor patients $(0-<18$ years old) accounted for less than one-third, while the number of elderly patients ( $\geq 65$ years old) was quite small. About a half of the patients were normal weight; underweight and obesity patients accounted for about one-fifth which was about a double of the overweight patients. Most of them were Han
TABLE 1: Demographics of the patients attending the nutrition clinical center $(\mathrm{n}=1014)$.

\begin{tabular}{lc}
\hline Demographics & No. (\%) of patients \\
\hline Sex & \\
Male & $483(47.6)$ \\
Female & $531(52.4)$ \\
Age (years) & \\
$0-<18$ & $281(27.7)$ \\
$18-64$ & $669(66.0)$ \\
$\geq 65$ & $64(6.3)$ \\
BMI categories & \\
Underweight & $222(21.9)$ \\
Normal weight & $455(44.9)$ \\
Overweight & $114(11.2)$ \\
Obesity & $223(22.0)$ \\
Region & \\
Urban & $947(93.4)$ \\
Rural & $67(6.6)$ \\
Education (years) & \\
0 & $119(11.7)$ \\
$0-6$ & $121(11.9)$ \\
$7-12$ & $609(60.1)$ \\
$>12$ & $165(16.3)$ \\
Ethnic group (nationalities) & \\
Han & $989(97.5)$ \\
Others & $25(2.5)$ \\
\hline
\end{tabular}

BMI, body mass index.

Chinese came from urban area and received education for 7-12 years. The demographics of the patients are shown in Table 1.

Majority of the patients $(41.9 \%)$ came to our clinical center for nutrition consultation of healthy dietary services, $32.1 \%$ for malnutrition, $6.7 \%$ for diabetes, $6.3 \%$ for neoplasms, $5.3 \%$ for digestive system diseases, and the last $7.6 \%$ for hypertension, hematologic diseases, thyroid diseases, and so on.

Healthy dietary services consisted of nutrition consultation of common diet $(34.7 \%)$, growth and development $(3.4 \%)$, maternal nutrition $(1.8 \%)$, preparation of pregnant $(0.7 \%)$, and others $(1.3 \%)$ such as preparation of college entrance examination and breastfeeding.

Malnutrition patients were divided into two groups: undernutrition (11.4\%) and overnutrition (20.7\%). The undernutrition group included thinness $(9.5 \%)$, child nutrition disorders (1.5\%), and deficiency diseases $(0.5 \%)$. The overnutrition group consisted of overweight $(6.9 \%)$ and obesity $(13.8 \%)$.

More minor patients came for healthy dietary services compared with the population $(\mathrm{P}<0.001)$, and, on the contrary, more adult patients came for malnutrition service, especially obesity $(\mathrm{P}<0.05)$, while more elderly patients came for consultation services of diabetes, neoplasms, digestive system diseases, and hypertension $(\mathrm{P}<0.05)$. The consultation spectrum of the patients is shown in Table 2. 


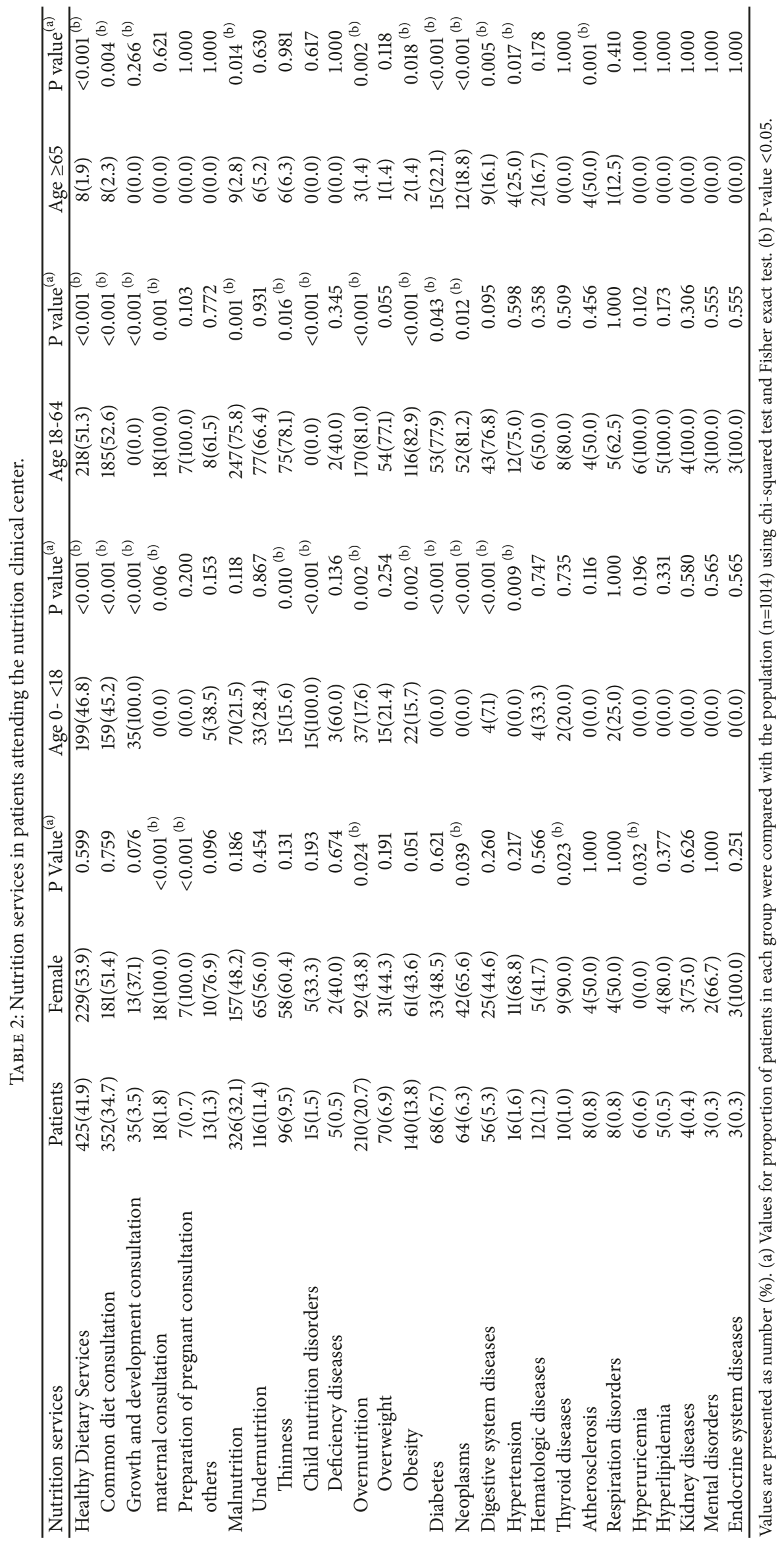


TABLE 3: Differences of demographics between main nutrition services.

\begin{tabular}{|c|c|c|c|c|c|c|}
\hline Variable & $\begin{array}{l}\text { Healthy Dietary } \\
\text { Services }(n=425)\end{array}$ & $\begin{array}{c}\text { Malnutrition } \\
(\mathrm{n}=326)\end{array}$ & P Value $^{(\mathrm{a})}$ & $\begin{array}{l}\text { Undernutrition } \\
\quad(\mathrm{n}=116)\end{array}$ & $\begin{array}{c}\text { Overnutrition } \\
(\mathrm{n}=210)\end{array}$ & P Value ${ }^{(a)}$ \\
\hline \multicolumn{7}{|l|}{ Sex } \\
\hline Female & 229 (53.9) & $157(48.2)$ & 0.120 & $65(56.0)$ & $92(43.8)$ & 0.086 \\
\hline \multicolumn{7}{|c|}{ Age (years) } \\
\hline $0-<18$ & $199(46.8)$ & $70(21.5)$ & $<0.001^{(\mathrm{b})}$ & $33(28.4)$ & $37(17.6)$ & $0.023^{(b)}$ \\
\hline $18-64$ & $218(51.3)$ & $247(75.8)$ & $<0.001^{\text {(b) }}$ & $77(66.4)$ & $170(81.0)$ & $0.003^{(b)}$ \\
\hline$\geq 65$ & $8(1.9)$ & $9(2.8)$ & 0.422 & $6(5.2)$ & $3(1.4)$ & 0.073 \\
\hline
\end{tabular}

Values are presented as number (\%). (a) Values for proportion of patients in each group were compared with each other using chi-squared test and Fisher exact test. (b) P-value $<0.05$.

No significant sex differences were found in the patients who came for nutrition services. About a half of the patients who came for healthy dietary services were minors, while most of the patients who came for malnutrition services were adults $(\mathrm{P}<0.001)$. More adult patients came due to overnutrition compared with undernutrition $(\mathrm{P}<0.01)$. Differences of demographics between main nutrition services are shown in Table 3.

\section{Discussion}

Along with the increasing incidence of nutrition-related chronic diseases such as obesity, hyperlipidemia, diabetes, cardio-cerebral vascular disease, cancer, and public awareness, there is a growing demand of public health nutrition service for nutrition-related issues in China in recent years.

A concerted effort was made in the mid-1980s to foster clinical nutrition in major hospitals throughout the country by programs directed at medical graduates and nursing and kitchen staff in China [9]. Traditionally, clinical nutritionists without prescription are only responsible for inpatient nutritional assessment and consultation as teamwork members with physicians of related specialties. Only a few comprehensive teaching hospitals established independent nutrition clinics as the new pattern of nutrition service, due to few physicians willing to accept nutrition professional training to work as nutrition physicians; from then on, the new public health nutrition service patterns were launched.

Our study showed that thousands of patients had been seen in the nutrition clinic as new cases in the past more than two years which indicated tremendous demand.

The population served by our nutrition outpatient clinic is extensive: age of patients ranged from 4 months to 92 years old. A large proportion of adult patients came to our clinic for nutrition services; on the contrary, minor patients only accounted for less than one-third and the number of elderly patients is quite small. However, population aging is becoming increasingly prominent in developing countries. The need for nutritional assessment and intervention is particularly crucial in elderly people due to the high incidence of chronic illness and malnutrition [10]. Similarly, the infant and young child population suffered more and more nutrition issues such as feeding practices, less breastfeeding, anemia, and protein energy malnutrition [11]. So, we believe that the awareness of nutrition issues for infant and young child and elderly population needs to be raised urgently.

Majority of the patients came to our clinic for healthy dietary services. The healthy dietary services is dietary nutrition consultation for all stages of healthy population without diseases. The main services were nutrition consultation of common diet, growth and development, maternal nutrition, and preparation of pregnancy.

The common diet nutrition consultation accounted for the largest proportion which may due to the unreasonable food consumption patterns. For adult, some studies indicated that Chinese food consumption patterns and eating and cooking behaviors changed dramatically with urbanization, for instance, the increasing consumption of energy from fats, the high level of sodium consumption, and the high sodium-potassium ratios [12]. For child population, many parents who came to our clinic complain that their children were picky eaters, in addition to high intakes of total fat and saturated fat, low intakes of fruits and vegetables were the most common problems. Some research indicated that the low intakes of fruits and vegetables are associated with inadequate intakes of vitamin $\mathrm{A}$, vitamin $\mathrm{C}$, and dietary fiber [13]. For infant and young child population, the early initiation of breastfeeding knowledge and practices of caregivers was poor. For example, most common problems are the insufficient time of exclusive breastfeeding and failing to add iron-rich or iron-fortified foods timely. Unreasonable food consumption patterns were considered as the reasons for many nutrition-related chronic diseases; therefore, the Chinese Nutrition Society revised the Food Guide Pagoda for Chinese Residents 2016 [14], after that the dietary counseling services provided followed.

Besides healthy dietary services, the second highest ranked nutrition service is about malnutrition. The prevalence of overweight and obesity is increasing worldwide and even developing countries are beginning to experience this trend in the last two decades [15]. It is common to see problems of underweight, stunting, and micronutrient deficiencies even with the prevalence of overweight and obesity increasing [16]. Our study showed that the number of overnutrition patients is significantly greater than undernutrition. As the rapid economic growth, overweight and obesity have become a major public health problem in China. Because of earlier experiences with long-term poverty and 
famine, the Chinese traditionally believe that fatness is a sign of happiness and abundance. These traditional beliefs have not kept pace with the socioeconomic changes that have led to a more secure food supply, overconsumption, and energy imbalance [17]. Therefore, we believe that the government should pay more attention to the overnutrition problems and take effective policy measures to curb the trend towards overweight and obesity in China. In addition, the proportion of overweight patients is significantly lower than obesity which may resulted from insufficient awareness of the early recognition of obesity and the harm to obesity.

Some other patients came to our clinic for various disease-related nutritional counseling. The main diseases were diabetes, neoplasms, digestive system diseases, and so on. Research has shown that diet is the cornerstone of treatment in diabetes and dietary advice should be tailored to the individual and their circumstances [18]. Nutritional management of the cancer patient cannot be ignored, given the well-established links between malnutrition and mortality, postoperative complications, toxicity of radiation, chemotherapy, and quality of life [19]. It is indicated that the comprehensive clinical medical background knowledge of nutrition physician is often required given the diversity of the clinical nutrition-related diseases in our clinic.

The limitation of our research is that it is a retrospective study in a single center. A nonrandom and unrepresentative sample limits the generalizability of our study findings. However, almost all patients in the local area came to our center. So we believe that our conclusions are credible and meaningful.

\section{Conclusion}

The present study provided important data of the new public health nutrition service pattern in China which has never been reported yet. It indicated the huge demand of nutrition outpatient clinic, especially nutrition consultation services of healthy diet and malnutrition; further studies about the validity of the new pattern in improving public health nutrition status are expected in the future.

\section{Data Availability}

The data used to support the findings of this study are available from the corresponding author upon request.

\section{Disclosure}

The funder had no role in the design, analysis, or writing of this article.

\section{Conflicts of Interest}

The authors declare that there are no conflicts of interest regarding the publication of this paper.

\section{Acknowledgments}

The authors would like to thank the staff of the Division of Nutrition, the Fourth Affiliated Hospital of Zhejiang
University School of Medicine, for providing necessary help for this project. This work is financially supported by Zhejiang Medical and Health Science and Technology Plan Project (Yunpeng Jin, Grant no. 2018KY873).

\section{References}

[1] L. Wang, Summary Report of China Nutrition and Health Survey, Medical Publishing House, Beijing, Peoples, 2002.

[2] L. i. LM, K. Q. Rao, and L. Z. Kong, "A description on the Chinese national nutrition and health survey in 2002," Zhonghua Liu Xing Bing Xue Za Zhi, vol. 26, no. 7, pp. 478-484, 2005.

[3] S. Du, B. Lu, F. Zhai, and B. M. Popkin, "A new stage of the nutrition transition in China," Public Health Nutrition, vol. 5, no. 1 A, pp. 169-174, 2002.

[4] B. M. Popkin, S. Du, F. Zhai, and B. Zhang, "Cohort profile: the China health and nutrition survey-monitoring and understanding socio-economic and health change in China, 1989-2011," International Journal of Epidemiology, vol. 39, no. 6, Article ID dyp322, pp. 1435-1440, 2010.

[5] T. Burrows, A. Patterson, A. Bacon et al., "Client satisfaction and weight loss outcomes of student centred dietetic outpatient clinics," Obesity Research \& Clinical Practice, vol. 7, no. 5, pp. e421-e430, 2013.

[6] W. Marcason, "Dietitian, dietician, or nutritionist?" Journal of the Academy of Nutrition and Dietetics, vol. 115, no. 3, p. 484, 2015.

[7] B.-F. Zhou, "Predictive values of body mass index and waist circumference for risk factors of certain related diseases in Chinese adults-study on optimal cut-off points of body mass index and waist circumference in Chinese adults," Biomedical and Environmental Sciences, vol. 15, no. 1, pp. 83-96, 2002.

[8] J. i. CY, J. L. Sun, and T. J. Chen, "Dynamic analysis on the prevalence of obesity and overweight school-age children and adolescents in recent 15 years in China," Zhonghua Liu Xing Bing Xue Za Zhi, vol. 25, pp. 103-108, 2004.

[9] M. L. Wahlqvist, “The rise of clinical North-East Asia," Asia Pacific Journal of Clinical Nutrition, vol. 25, pp. 437-443, 2016.

[10] T. Pepersack, "Nutritional problems in the elderly," Acta clinica Belgica, vol. 64, no. 5, pp. 85-91, 2009.

[11] L. T. Cavalli-Sforza, "Public health\&nutrition in the asia-pacific: reflections on a quarter century," Asia Pacific Journal of Clinical Nutrition, vol. 24, no. 1, pp. 1-9, 2015.

[12] F. Y. Zhai, S. F. Du, Z. H. Wang, J. G. Zhang, W. W. Du, and B. M. Popkin, "Dynamics of the Chinese diet and the role of urbanicity, 1991-2011," Obesity Reviews, vol. 15, supplement 1, pp. 16-26, 2014.

[13] B. A. Dennison, H. L. Rockwell, and S. L. Baker, "Fruit and vegetable intake in young children," Journal of the American College of Nutrition, vol. 17, no. 2, pp. 371-378, 1998.

[14] "Dietary Guidelines and the Food Guide Pagoda," Journal of the Academy of Nutrition and Dietetics, vol. 100, no. 8, pp. 886-887, 2000.

[15] Y.-X. Zhang, M. Lin, and G.-Z. Sun, "The double burden of overweight and thinneß among children and adolescents in Shandong China," International Journal of Cardiology, vol. 184, no. 1, pp. 380-381, 2015.

[16] H. Wang and F. Zhai, "Programme and policy options for preventing obesity in China," Obesity Reviews, vol. 14, pp. 134-140, 2013. 
[17] Y. Wang, C. Monteiro, and B. M. Popkin, “Trends of obesity and underweight in older children and adolescents in the United States, Brazil, China, and Russia," American Journal of Clinical Nutrition, vol. 75, no. 6, pp. 971-977, 2002.

[18] S. Hughes, "Diabetes: support for those at risk of malnutrition in the community," British Journal of Community Nursing, vol. 17, no. 11, pp. 529-534, 2012.

[19] S. Antoun and V. Baracos, "Malnutrition in cancer patient: When to have a specialized consultation?" Bulletin du Cancer, vol. 96, no. 5, pp. 615-623, 2009. 


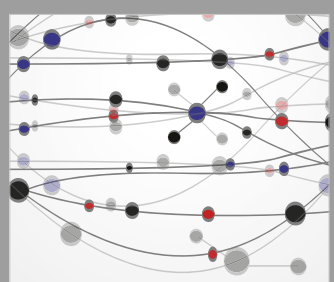

The Scientific World Journal
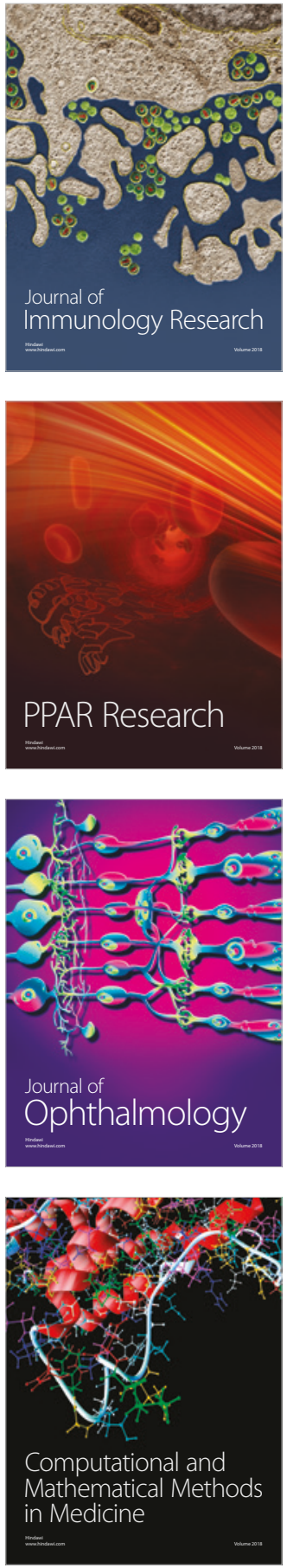

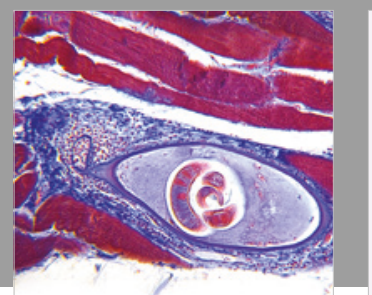

Gastroenterology Research and Practice

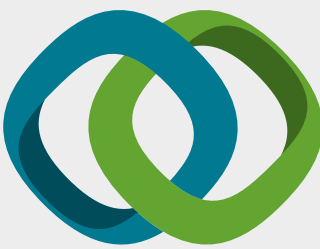

\section{Hindawi}

Submit your manuscripts at

www.hindawi.com
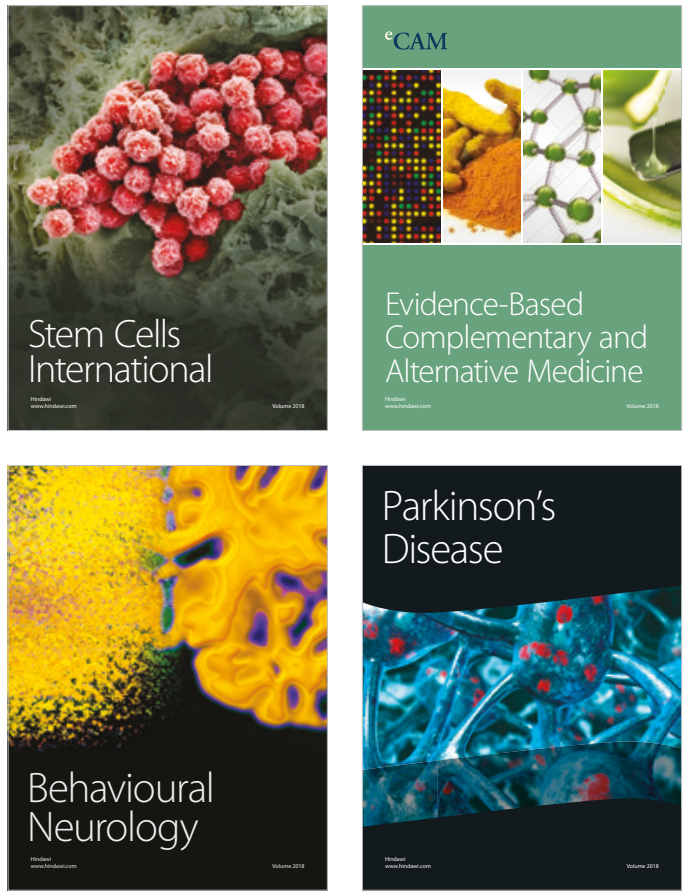

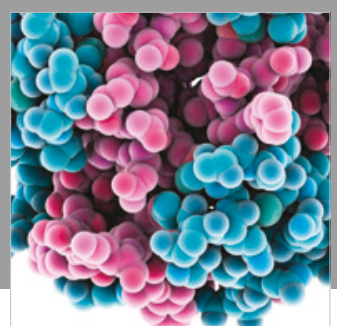

ournal of

Diabetes Research

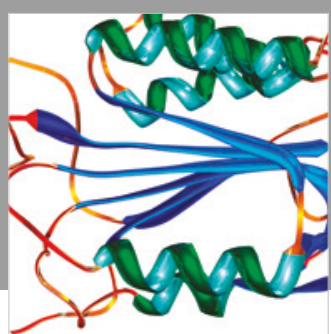

Disease Markers
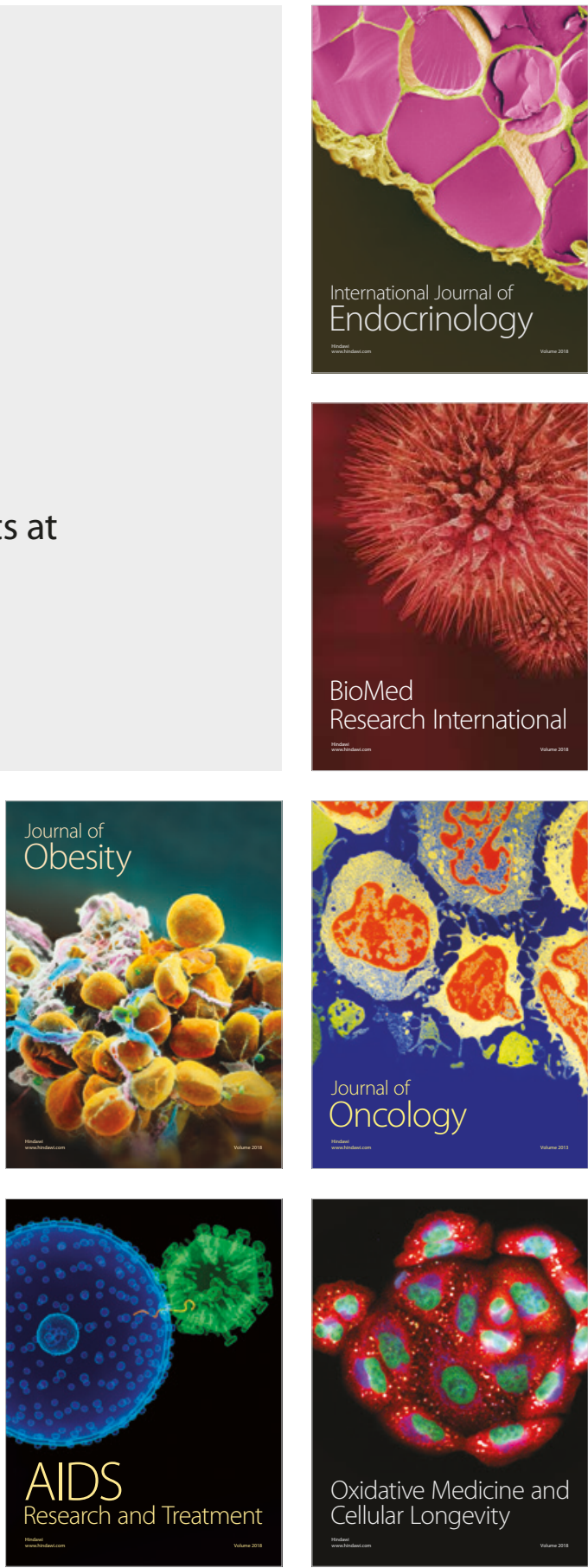